\title{
Spin-resolved second-order correlation energy of the two-dimensional uniform electron gas
}

\author{
Michael Seidl, Institute of Theoretical Physics, University of Regensburg, \\ D-93040 Regensburg, Germany
}

\begin{abstract}
For the two-dimensional electron gas, the exact high-density limit of the correlation energy is evaluated here numerically for all values of the spin polarization. The result is spinresolved into $\uparrow \uparrow, \uparrow \downarrow$, and $\downarrow \downarrow$ contributions and parametrized analytically. Interaction-strength interpolation yields a simple model (LSD) for the correlation energy at finite densities.
\end{abstract}

In recent years, two-dimensional (2D) electron systems have become the subject of extensive research [1]. The 2D version of density functional theory (DFT) has proven particularly successful in studying quantum dots [2, 3, 4]. The local spin-density approximation (LSD) of DFT requires the correlation energy of the spin-polarized uniform electron gas. This quantity in 2D is known accurately for a wide range of densities and spin polarizations from fixed-node diffusion Monte Carlo simulations [5]. Its high-density limit is known exactly in terms of six-dimensional momentum-space integrals [6]. Resolved into contributions due to $\uparrow \uparrow, \uparrow \downarrow$, and $\downarrow \downarrow$ excitation electron pairs, these integrals are evaluated here numerically. The analytical parametrization of the results, Eqs. (16) and (17) below, is a crucial ingredient for the construction of the spin-resolved correlation energy at finite densities, performed recently for the 3D electron gas [7]. It is also required for studying the magnetic response of the spin-polarized 2D electron gas [8, 9]. Generally, it provides a fundamental test for numerical parametrizations of the correlation energy [5].

In the $2 \mathrm{D}$ uniform electron gas, the electrons are moving on a plane at uniform density $\rho=\left[\pi\left(r_{s} a_{B}\right)^{2}\right]^{-1}$, where $a_{B}=0.529 \AA$ is the Bohr radius and $r_{s}$ is the dimensionless density parameter (Seitz radius). We consider lowest-energy states with a given spin polarization

$$
\zeta \equiv \frac{\rho_{\uparrow}-\rho_{\downarrow}}{\rho}
$$

where $\rho_{\uparrow}$ and $\rho_{\downarrow} \equiv \rho-\rho_{\uparrow}$, respectively, are the (uniform) densities of spin-up and spin-down electrons. Including a neutralizing positive background, the total energy per electron is a unique function of the dimensionless parameters $r_{s}$ and $\zeta$,

$$
e_{t o t}\left(r_{s}, \zeta\right)=t_{s}\left(r_{s}, \zeta\right)+e_{x}\left(r_{s}, \zeta\right)+e_{c}\left(r_{s}, \zeta\right) .
$$

The non-interacting kinetic and exchange energies,

$$
t_{s}\left(r_{s}, \zeta\right)=\frac{1+\zeta^{2}}{2} \frac{1}{r_{s}^{2}}, \quad e_{x}\left(r_{s}, \zeta\right)=-\frac{4 \sqrt{2}}{3 \pi} \frac{(1+\zeta)^{3 / 2}+(1-\zeta)^{3 / 2}}{2} \frac{1}{r_{s}}
$$


(all energies are given in units of $1 \mathrm{Ha} \equiv e^{2} / a_{B}=27.21 \mathrm{eV}$ in the following), may be understood as the 0th- and the 1st-order terms of a perturbation expansion for the electron-electron interaction (where $r_{s}$ turns out to be the expansion parameter).

The remaining correlation energy in Eq. (2) appears to have the perturbation (highdensity) expansion [10, 11]

$$
e_{c}\left(r_{s}, \zeta\right)=\sum_{n=0}^{\infty}\left[a_{n}(\zeta) \ln \left(r_{s}\right)+b_{n}(\zeta)\right] r_{s}^{n} \quad\left(r_{s} \ll 1\right) .
$$

For the $2 \mathrm{D}$ electron gas (but not for the $3 \mathrm{D}$ one), the first coefficient vanishes, $a_{0}(\zeta) \equiv 0$. Consequently, the second-order $(n=0)$ term is $e_{c}^{(2)}(\zeta) \equiv b_{0}(\zeta)$, representing the highdensity $\left(r_{s} \rightarrow 0\right)$ limit of $e_{c}\left(r_{s}, \zeta\right)$. It can be split into an exchange (" $2 b$ ") and a ringdiagram (" $2 r$ ") term [6],

$$
e_{c}^{(2)}(\zeta)=e_{c}^{(2 b)}+e_{c}^{(2 r)}(\zeta)
$$

The exchange term has only equal-spins contributions, $e_{c}^{(2 b)}=e_{c \uparrow \uparrow}^{(2 b)}(\zeta)+e_{c \downarrow \downarrow}^{(2 b)}(\zeta)$, given by the $\delta_{\sigma_{1} \sigma_{2}}$ term of Eq. (14) in Ref. [6] (we choose the $k_{x}$ axis in the direction of $\mathbf{q}$ ),

$$
e_{c, \sigma \sigma}^{(2 b)}(\zeta)=\frac{1}{8 \pi^{2}} \int_{0}^{\infty} \frac{d q}{q} \int_{A\left[\kappa_{\sigma}(\zeta), q\right]} d^{2} k_{1} \int_{A\left[\kappa_{\sigma}(\zeta), q\right]} d^{2} k_{2} \frac{1}{\left|q \mathbf{e}_{x}+\mathbf{k}_{1}+\mathbf{k}_{2}\right|} \frac{1}{q+k_{1 x}+k_{2 x}}
$$

Here, $q, \mathbf{k}_{1}$, and $\mathbf{k}_{2}$ are dimensionless, $\sigma \in\{\uparrow, \downarrow\}$, and the domain of the 2D integrals is

$$
A[\kappa, q] \equiv\left\{\mathbf{k} \in \mathrm{R}^{2}|| \mathbf{k}|<\kappa,| \mathbf{k}+q \mathbf{e}_{x} \mid>\kappa\right\}, \quad \kappa_{\sigma}(\zeta) \equiv[1+\operatorname{sgn}(\sigma) \zeta]^{1 / 2}
$$

$\left[\kappa_{\sigma}(\zeta)\right.$ is the Fermi wave vector for spin- $\sigma$ electrons in units of its value at $\zeta=0$.] Scaling the integration variables by some constant $\kappa, q=\kappa Q$ and $\mathbf{k}=\kappa \mathbf{K}$, we have generally

$$
\int_{0}^{\infty} \frac{d q}{q} \int_{A[\kappa, q]} d^{2} k f(q, \mathbf{k})=\kappa^{2} \int_{0}^{\infty} \frac{d Q}{Q} \int_{A[1, Q]} d^{2} K f(\kappa q, \kappa \mathbf{K}) .
$$

Applying this rule to the integrals in Eq. (6) , we find [6]

$$
e_{c, \sigma \sigma}^{(2 b)}(\zeta)=[1+\operatorname{sgn}(\sigma) \zeta] J^{(2 b)}
$$

Consequently [6], the full second-order exchange term $e_{c}^{(2 b)}=e_{c \uparrow \uparrow}^{(2 b)}(\zeta)+e_{c \downarrow \downarrow}^{(2 b)}(\zeta) \equiv 2 J^{(2 b)}$ is $\zeta$-independent. A Monte Carlo integration yields

$$
J^{(2 b)} \equiv e_{c \uparrow \uparrow}^{(2 b)}(0)=(57.15 \pm 0.05) \mathrm{mHa} \quad\left(1 \mathrm{mHa}=10^{-3} \mathrm{Ha}\right)
$$


The ring-diagram term $e_{c}^{(2 r)}(\zeta)$ is the remaining part of expression (14) in Ref. [6], with the contributions

$$
e_{c, \sigma_{1} \sigma_{2}}^{(2 r)}(\zeta)=-\frac{1}{8 \pi^{2}} \int_{0}^{\infty} \frac{d q}{q^{2}} \int_{A\left[\kappa_{\sigma_{1}}(\zeta), q\right]} d^{2} k_{1} \int_{A\left[\kappa_{\sigma_{2}}(\zeta), q\right]} d^{2} k_{2} \frac{1}{q+k_{1 x}+k_{2 x}} .
$$

The equal-spins terms $\left(\sigma_{1}=\sigma_{2}\right)$ can be treated in the same way as the integral (6)),

$$
e_{c, \sigma \sigma}^{(2 r)}(\zeta)=-[1+\operatorname{sgn}(\sigma) \zeta] J^{(2 r)}, \quad J^{(2 r)}=(76.69 \pm 0.03) \mathrm{mHa} .
$$

The only non-trivial $\zeta$-dependence is in the opposite-spins term $e_{c \uparrow \downarrow}^{(2 r)}(\zeta) \equiv e_{c \downarrow \uparrow}^{(2 r)}(\zeta)$,

$$
e_{c \uparrow \downarrow}^{(2 r)}(\zeta)=e_{c \uparrow \downarrow}^{(2 r)}(0)[1-f(\zeta)]
$$

By definition, $f(0)=0$, and, since $A\left[\kappa_{\downarrow}(1), q\right]=\emptyset, f(1)=1$. Moreover, $e_{c \uparrow \downarrow}^{(2 r)}(0)=-J^{(2 r)}$. When the results of a Monte Carlo evaluation of $f(\zeta)$ at different values of $\zeta$ are compared with the functions $f_{\alpha}(\zeta) \equiv\left[(1+\zeta)^{\alpha}+(1-\zeta)^{\alpha}-2\right] /\left(2^{\alpha}-2\right)$, particularly good agreement (specially for $\zeta \rightarrow 0$ and $\zeta \rightarrow 1$ ) is found in the limit $\alpha \rightarrow 1$ (Fig. 1a),

$$
f(\zeta)=f_{1}(\zeta)+\delta f(\zeta), \quad f_{1}(\zeta) \equiv \frac{(1+\zeta) \ln (1+\zeta)+(1-\zeta) \ln (1-\zeta)}{2 \ln 2}
$$

[Note that $f_{\alpha}(\zeta)$ also represents the $\zeta$-dependence of $t_{s}(\alpha=2)$ and $e_{x}\left(\alpha=\frac{3}{2}\right)$ in Eq. (3).] The small deviation $\delta f(\zeta)$ is accurately fitted by a polynomial (Fig. 1b)

$$
\delta f(\zeta) \approx 0.0636 \zeta^{2}-0.1024 \zeta^{4}+0.0389 \zeta^{6}
$$

The small minimum of $\delta f(\zeta)$ indicated by the numerical data (dots in Fig. $1 \mathrm{~b}$ ) at $\zeta \approx 0.98$ is probably real, since a similar peculiarity is observed for the $3 \mathrm{D}$ electron gas (see the inset in Fig. 1 of Ref. [12]).

In summary, the second-order correlation energy $e_{c}^{(2)}(\zeta)=e_{c}^{(2 b)}+e_{c}^{(2 r)}(\zeta)$ is

$$
e_{c}^{(2)}(\zeta) \equiv e_{c \uparrow \uparrow}^{(2)}(\zeta)+2 e_{c \uparrow \downarrow}^{(2)}(\zeta)+e_{c \downarrow \downarrow}^{(2)}(\zeta)=[153.38 f(\zeta)-192.46] \mathrm{mHa},
$$

where $f(\zeta)$ is given by Eqs. (14) and (15). The spin resolution is fixed by

$$
e_{c \uparrow \uparrow}^{(2)}(\zeta) \equiv e_{c \downarrow \downarrow}^{(2)}(-\zeta)=-(1+\zeta) \times 19.54 \mathrm{mHa} .
$$

$e_{c}^{(2)}(\zeta) \equiv e_{c}(0, \zeta)$ is the high-density limit of the general correlation energy $e_{c}\left(r_{s}, \zeta\right)$. To illustrate the relevance of this limit for finite densities $\left(r_{s}>0\right)$, the present result can be used in the interaction-strength interpolation (ISI) of Ref. [13. This approach does not require the higher-order $(n \geq 1)$ terms of the expansion (4) (which is expected 
to have only a finite radius of convergence). Instead, information beyond the second order is taken from the low-density (strong-interaction or Wigner-crystal) limit of the exchange-correlation energy $e_{x c} \equiv e_{x}+e_{c}$ (per electron),

$$
e_{x c}\left(r_{s}, \zeta\right) \rightarrow \frac{a_{\infty}}{r_{s}}+\frac{b_{\infty}}{r_{s}^{3 / 2}} \quad\left(r_{s} \rightarrow \infty\right)
$$

The coefficients [14] $a_{\infty} \approx-1.1061$ and $b_{\infty} \approx \frac{1}{2}$ are independent of $\zeta$, since any spatial overlap between two electrons is strongly suppressed in this limit, no matter whether their spins are parallel or not [15]. The resulting ISI expression for the exchange-correlation energy at finite densities reads [13]

$$
e_{x c}^{I S I}\left(r_{s}, \zeta\right)=\frac{a_{\infty}}{r_{s}}+\frac{2 X}{Y}\left[(1+Y)^{1 / 2}-1-Z \ln \left(\frac{(1+Y)^{1 / 2}+Z}{1+Z}\right)\right] .
$$

Using $b_{\infty}=\frac{1}{2}$ and writing $e_{x}\left(r_{s}, \zeta\right)=c_{x}(\zeta) / r_{s}$, we have explicitly [13]

$$
\begin{aligned}
X\left(r_{s}, \zeta\right) & =\frac{-b_{0}(\zeta)}{\left[c_{x}(\zeta)-a_{\infty}\right]^{2}} \frac{1}{r_{s}}, \\
Y\left(r_{s}, \zeta\right) & =\frac{4 b_{0}(\zeta)^{2}}{\left[c_{x}(\zeta)-a_{\infty}\right]^{4}} r_{s}, \\
Z(\zeta) & =\frac{-b_{0}(\zeta)}{\left[c_{x}(\zeta)-a_{\infty}\right]^{3}}-1 .
\end{aligned}
$$

Eq. (19) provides a simple explicit LSD,

$$
E_{x c}^{L S D}\left[\rho_{\uparrow}, \rho_{\downarrow}\right]=\int d^{2} r \rho(\mathbf{r}) e_{x c}^{I S I}\left(r_{s}(\mathbf{r}), \zeta(\mathbf{r})\right),
$$

for treating arbitrary 2D electron systems (also finite ones such as quantum dots) by the Kohn-Sham Equations of DFT. In Eq. (21),$r_{s}(\mathbf{r})=a_{B}^{-1}[\pi \rho(\mathbf{r})]^{-1 / 2}$ and $\zeta(\mathbf{r})=\left[\rho_{\uparrow}(\mathbf{r})-\right.$ $\left.\rho_{\downarrow}(\mathbf{r})\right] / \rho(\mathbf{r})$.

In Fig. 2a, the ISI prediction $e_{c}^{I S I}\left(r_{s}, \zeta\right)=e_{x c}^{I S I}-e_{x}$ for the correlation energy of the unpolarized uniform electron gas $(\zeta=0)$ is compared with the accurate parametrization of the fixed-node diffusion Monte Carlo results in Ref. [5]. $e_{c}^{I S I}$ differs slightly from the latter by up to $4 \%$. This mild deviation might be cured by including in the ISI a simple model for the next-order coefficient of expansion (4) [16. In the high-density limit $\left(r_{s} \rightarrow 0\right)$, however, where the present result is exact, the parametrization in Ref. [5] has for $0.7<\zeta<0.95$ a small positive deviation [5], shown in Fig. 2 b. 


\section{Figure captions:}

Fig. 1. (a) Numerical results (dots) for the function $f(\zeta)$ of Eq. (13) obtained by Monte Carlo integrations of expression (11) (with $\sigma_{1} \sigma_{2}=\uparrow \downarrow$ ) at selected values of $\zeta$. The analytical function $f_{1}(\zeta)$ of Eq. (14) is plotted as a dashed curve. The solid curve represents the accurate fit $f_{1}(\zeta)+\delta f(\zeta)$, using Eq. (15) for $\delta f(\zeta)$. (b) The fit (15) (solid curve) compared to the true deviation (dots) of the Monte-Carlo-integration results from $f_{1}(\zeta)$.

Fig. 2. The correlation energy of Ref. [5] (dotted curves) versus the present ISI results (solid curves).

\section{References}

[1] E. Abrahams, S. V. Kravchenko, and M. P. Sarachik, Rev. Mod. Phys. 73, 251 (2001).

[2] S.M. Reimann and M. Manninen, Rev. Mod. Phys. 74, 1283 (2002).

[3] H. Jiang, H.U. Baranger, and W. Yang, Phys. Rev. B 68, 165337 (2003).

[4] H. Saarikoski, E. Räsänen, S. Siljamäki, A. Harju, M.J. Puska, R.M. Nieminen, Phys. Rev. B 67, 205327 (2003).

[5] C. Attaccalite, S. Moroni, P. Gori-Giorgi, and G. B. Bachelet, Phys. Rev. Lett. 88, 256601 (2002).

[6] A.K. Rajagopal and J.C. Kimball, Phys. Rev. B 15, 2819 (1977).

[7] P. Gori-Giorgi and J.P. Perdew, Phys. Rev. B (to appear; cf. cond-mat/0305250).

[8] J. Moreno and D.C. Marinescu, Phys. Rev. B 68, 195210 (2003)

[9] M. Polini and M.P. Tosi, Phys. Rev. B 63, 045118 (2001).

[10] W. Macke, Z. Naturforsch. A 5A, 192 (1950).

[11] W.J. Carr and A.A. Maradudin, Phys. Rev. 133, A371 (1964).

[12] G.G. Hoffman, Phys. Rev. B 45, 8730 (1992).

[13] M. Seidl, J.P. Perdew, and S. Kurth, Phys. Rev. Lett. 84, 5070 (2000).

[14] L. Bonsall and A.A. Maradudin, Phys. Rev. B 15, 1959 (1977).

[15] M. Seidl, J.P. Perdew, and S. Kurth, Phys. Rev. A 62, 012502 (2000).

[16] M. Seidl and J.P. Perdew (unpublished). 

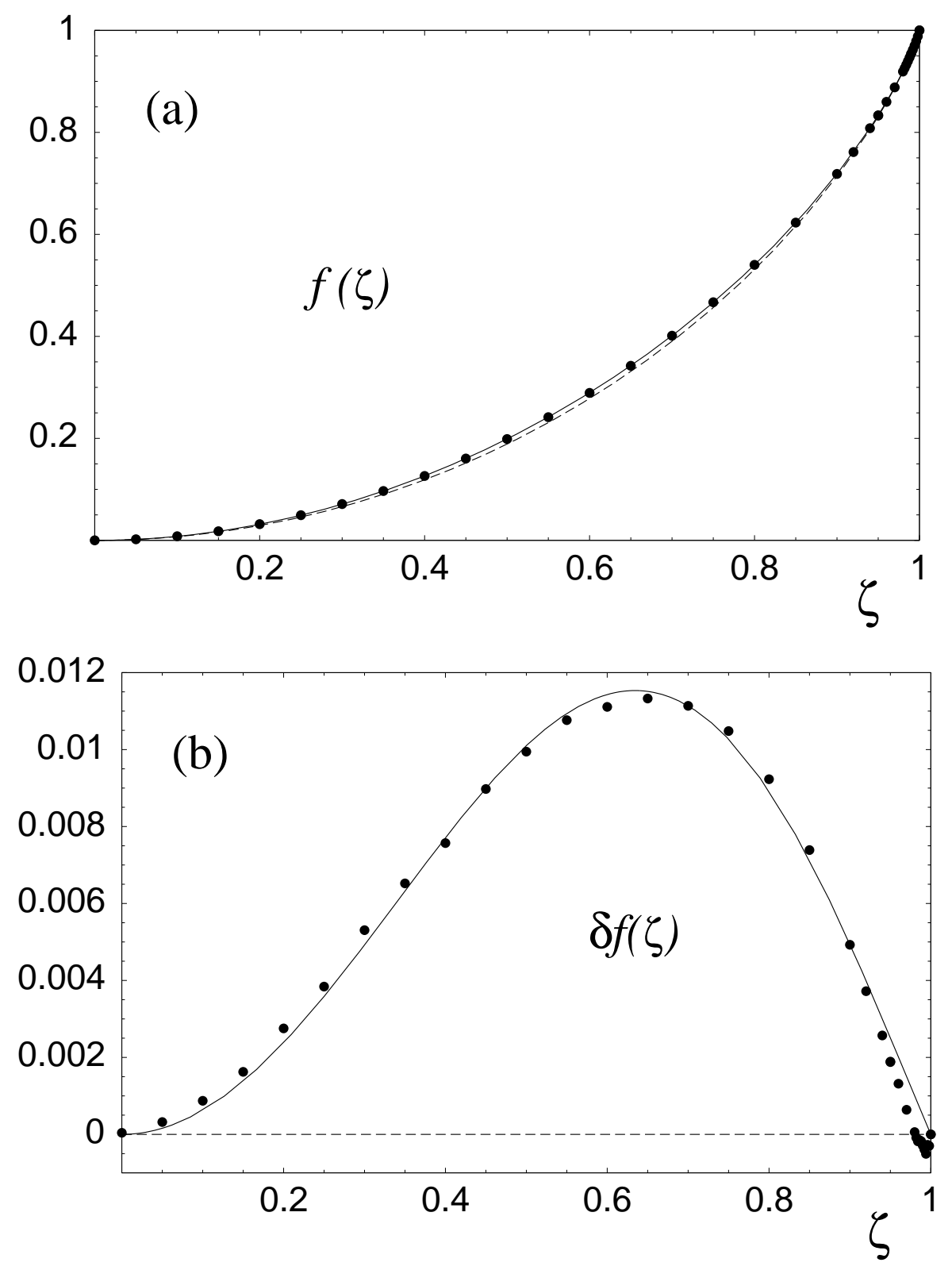

Figure 1: (a) Numerical results (dots) for the function $f(\zeta)$ of Eq. (13) obtained by Monte Carlo integrations of expression (11) (with $\left.\sigma_{1} \sigma_{2}=\uparrow \downarrow\right)$ at selected values of $\zeta$. The analytical function $f_{1}(\zeta)$ of Eq. (14) is plotted as a dashed curve. The solid curve represents the accurate fit $f_{1}(\zeta)+\delta f(\zeta)$, using Eq. (15) for $\delta f(\zeta)$. (b) The fit (15) (solid curve) compared to the deviation (dots) of the MonteCarlo-integration results from $f_{1}(\zeta)$. 

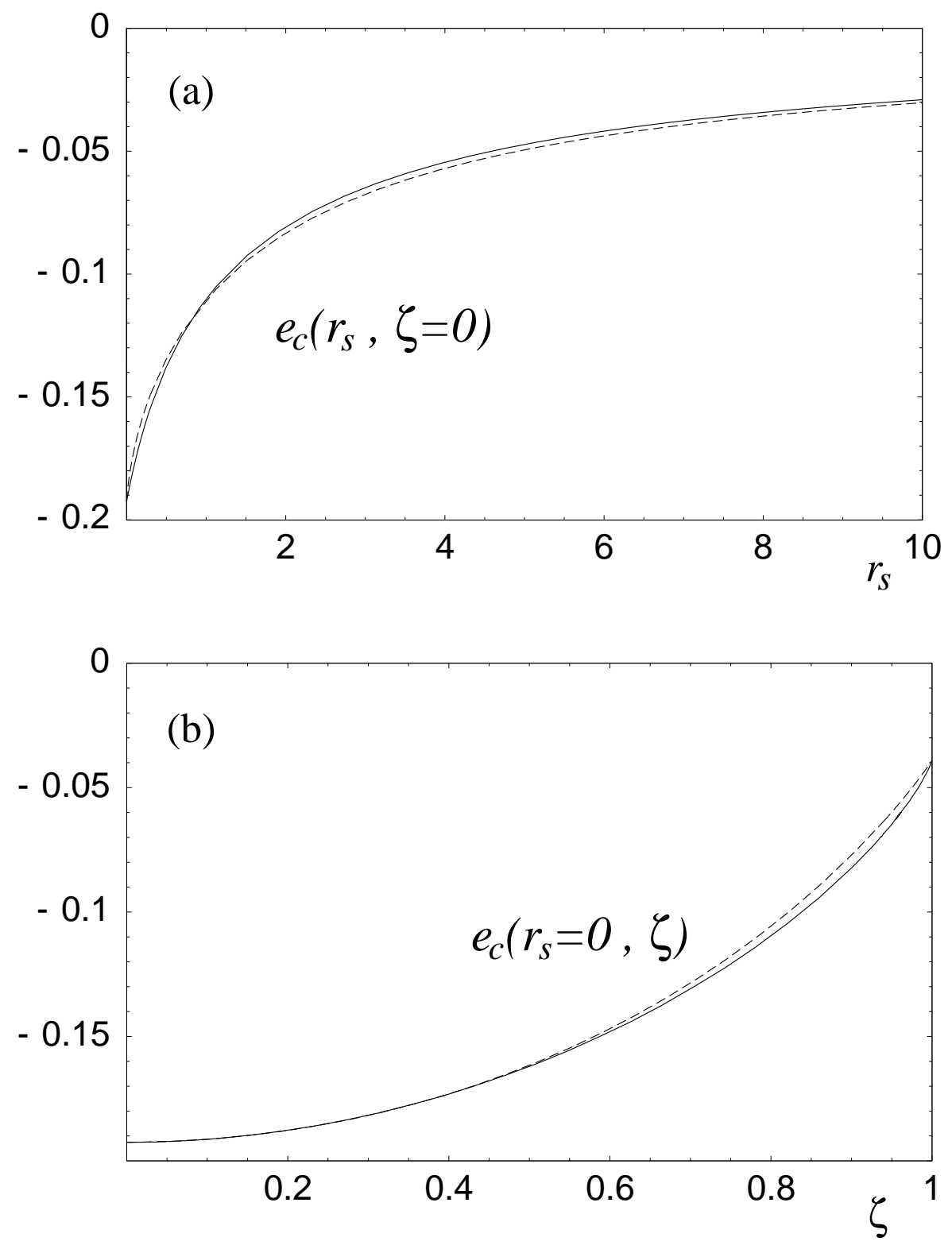

Figure 2: The correlation energy of Ref. [5] (dotted curves) versus the present ISI results (solid curves). 